\title{
Discovery of a nanodiamond-rich layer in the Greenland ice sheet
}

\author{
Andrei V. KURBATOV, ${ }^{1}$ Paul A. MAYEWSKI, ${ }^{1}$ Jorgen P. STEFFENSEN, ${ }^{2}$ Allen WEST, ${ }^{3}$ \\ Douglas J. KENNETT, ${ }^{4}$ James P. KENNETT, ${ }^{5}$ Ted E. BUNCH, ${ }^{6}$ Mike HANDLEY, ${ }^{1}$ \\ Douglas S. INTRONE, ${ }^{1}$ Shane S. QUE HEE, ${ }^{7}$ Christopher MERCER, ${ }^{8}$ Marilee SELLERS, ${ }^{9}$ \\ Feng SHEN, ${ }^{10}$ Sharon B. SNEED, ${ }^{1}$ James C.WEAVER, ${ }^{11}$ James H. WITTKE, ${ }^{6}$ \\ Thomas W. STAFFORD, $\mathrm{Jr}^{12}{ }^{12}$ John J. DONOVAN, ${ }^{13}$ Sujing XIE, ${ }^{13}$ Joshua J. RAZINK, ${ }^{14}$ \\ Adrienne STICH, ${ }^{15}$ Charles R. KINZIE, ${ }^{15}$ Wendy S. WOLBACH ${ }^{15}$ \\ ${ }^{1}$ Climate Change Institute, University of Maine, 303 Bryand Global Sciences Center, Orono, Maine 04469-5790, USA \\ E-mail: akurbatov@maine.edu \\ ${ }^{2}$ Centre for Ice and Climate, Niels Bohr Institute, University of Copenhagen, Juliane Maries Vej 30, DK-2100 Copenhagen, \\ Denmark \\ ${ }^{3}$ GeoScience Consulting, Dewey, Arizona 86327, USA \\ ${ }^{4}$ Department of Anthropology, University of Oregon, Eugene, Oregon 97403-1272, USA \\ ${ }^{5}$ Department of Earth Science, University of California, Santa Barbara, California 93106-5131, USA \\ ${ }^{6}$ Department of Geology, Northern Arizona University, Flagstaff, Arizona 86011-4099, USA \\ ${ }^{7}$ Department of Environmental Health Sciences/Center for Occupational and Environmental Health, University of California, \\ Los Angeles, California 90095-1772, USA \\ ${ }^{8}$ National Institute for Materials Science, 1-2-1 Sengen, Tsukuba 305-0047, Japan \\ ${ }^{9}$ Imaging and Histology Core Facility, Northern Arizona University, Flagstaff, Arizona 86011, USA \\ ${ }^{10}$ FEI Company, 5350 NE Dawson Creek Drive, Hillsboro, Oregon 97124-5793, USA \\ ${ }^{11}$ Department of Physics, University of California, Santa Barbara, California 93106-5131, USA \\ ${ }^{12}$ Stafford Research Laboratories, Inc., 200 Acadia Avenue, Lafayette, Colorado 80026-1845, USA \\ ${ }^{13}$ CAMCOR High Resolution and MicroAnalytical Facilities, University of Oregon, Eugene, Oregon 97403-1272, USA \\ ${ }^{14}$ Department of Chemistry, University of Oregon, Eugene, Oregon 97403-1272, USA \\ ${ }^{15}$ Department of Chemistry, DePaul University, Chicago, Illinois 60614, USA
}

\begin{abstract}
We report the discovery in the Greenland ice sheet of a discrete layer of free nanodiamonds (NDs) in very high abundances, implying most likely either an unprecedented influx of extraterrestrial (ET) material or a cosmic impact event that occurred after the last glacial episode. From that layer, we extracted n-diamonds and hexagonal diamonds (lonsdaleite), an accepted ET impact indicator, at abundances of up to about $5 \times 10^{6}$ times background levels in adjacent younger and older ice. The NDs in the concentrated layer are rounded, suggesting they most likely formed during a cosmic impact through some process similar to carbon-vapor deposition or high-explosive detonation. This morphology has not been reported previously in cosmic material, but has been observed in terrestrial impact material. This is the first highly enriched, discrete layer of NDs observed in glacial ice anywhere, and its presence indicates that ice caps are important archives of ET events of varying magnitudes. Using a preliminary ice chronology based on oxygen isotopes and dust stratigraphy, the ND-rich layer appears to be coeval with ND abundance peaks reported at numerous North American sites in a sedimentary layer, the Younger Dryas boundary layer (YDB), dating to $12.9 \pm 0.1 \mathrm{ka}$. However, more investigation is needed to confirm this association.
\end{abstract}

\section{INTRODUCTION}

Recently scientists reported an abundance peak in nanodiamonds (NDs) at multiple locations across North America that is restricted to a thin sediment layer, the Younger Dryas boundary layer (YDB), which dates to the Younger Dryas (YD) onset at 12.9 $\pm 0.1 \mathrm{ka}$ (Firestone and others, 2007; Kennett and others, 2009a). A peak in hexagonal diamonds (lonsdaleite) was also reported in the YDB at Arlington Canyon, California, USA (Kennett and others, 2009b). The only known explanation for terrestrial lonsdaleite is by arrival inside extraterrestrial objects and/or by impact of such objects with the Earth's surface (DeCarli and others, 2002). This discovery begged a prediction that a coeval layer with high ND concentrations should be preserved in the Greenland ice sheet. No layers of NDs have previously been reported from the Greenland ice sheet, or any other form of glacial ice, although Yates and others (1992) reported finding diamond concentrations in Greenland cryoconite holes, which are melt depressions in surface ice that contain micrometeoritic material. However, those diamonds were not in a discrete layer and were found embedded in metal-alloy cosmic grains, rather than being free in the ice. Consequently, we conducted a pilot investigation on a section of the Greenland ice sheet that potentially spans the last deglacial in search of a layer containing an abundance of free NDs that might be coeval with that of the YDB, a study that included both field and laboratory components. The purpose of this contribution is to describe this pilot experiment and its limitations, to present our results and to suggest future work related to the discovery of NDs in this sequence. 


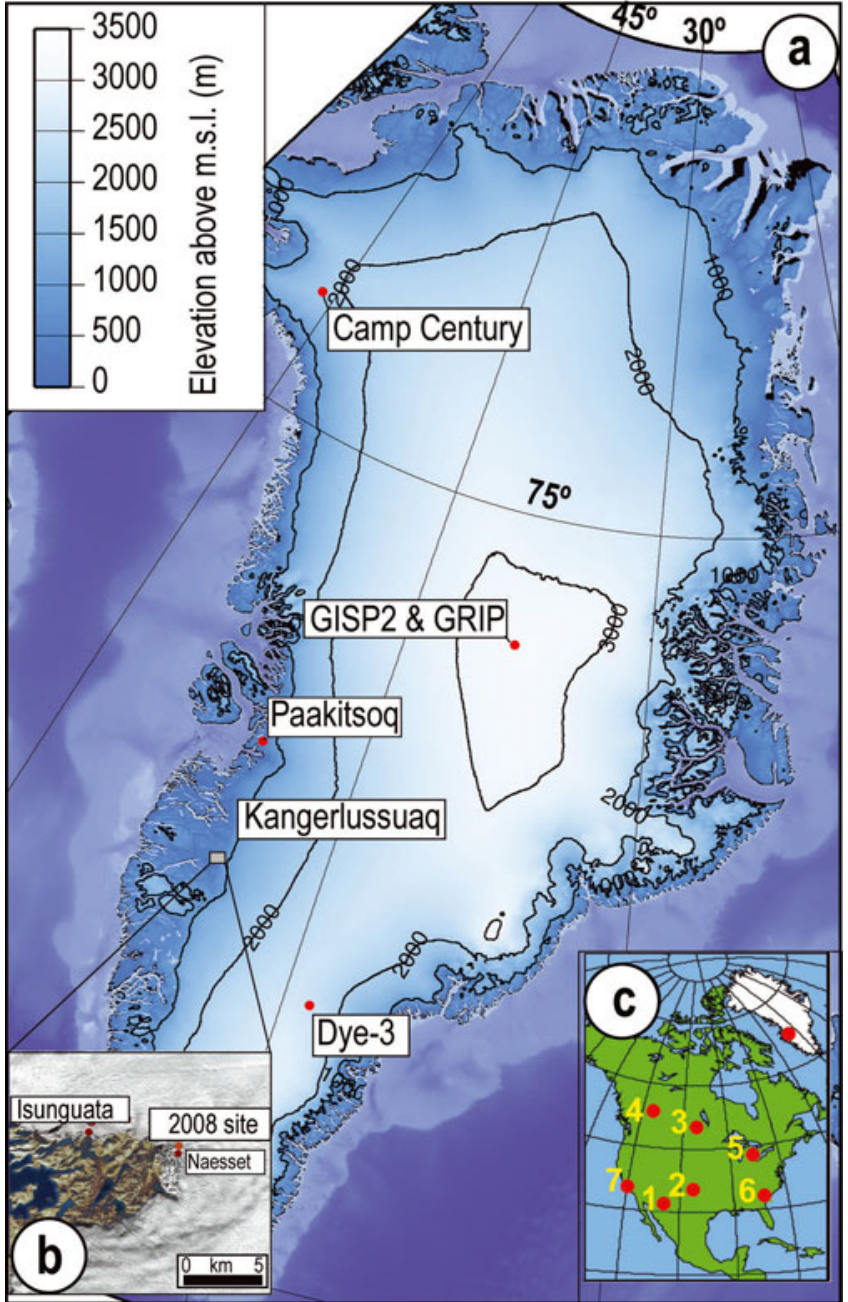

Fig. 1. Distribution of impact markers at sites in the Northern Hemisphere. (a) The Greenland ice sheet showing ice-core drilling sites, elevation contours and, with inset (b), location of the 2008 sampling site and the closest site, Næsset, with stable-isotope ratios reported by Reeh and others (2002). The closest source region for the ice transported to the margin near Kangerlussuaq is the region near Dye-3. (c) Sampling site in Greenland relative to North American sites with known YDB nanodiamond material: (1) Murray Springs, Arizona, USA: 200 ppb; (2) Bull Creek, Oklahomka, USA: 100 ppb; (3) Lake Hind, Manitoba, Canada: 70 ppb; (4) Chobot, Alberta, Canada: $10 \mathrm{ppb}$; (5) Gainey, Michigan, USA: $3700 \mathrm{ppb;}$ (6) Topper, South Carolina, USA: 108 ppb; (7) Arlington Canyon, California, USA: 1340 ppb (from Kennett and others, 2009a,b).

\section{METHODS AND SITE SELECTION}

Ice samples of large volume were necessary to conduct these investigations, and sufficient ice from deep cores (e.g. Greenland Ice Sheet Project 2 (GISP2), Greenland Icecore Project (GRIP) or NorthGRIP) are unavailable. Consequently, we followed the earlier approach of Reeh and others (2002) and Petrenko and others (2006), who sampled the ice margin to obtain a stratigraphic sequence over the interval from the last deglacial through the Holocene. In cooperation with PBS Nova documentary productions, we conducted fieldwork in late 2008 during which several authors (A.V.K., P.A.M. and J.P.S.) sampled at a margin site east of Kangerlussuaq, West Greenland, $\sim 1 \mathrm{~km}$ inland from the ice margin (Fig. 1). Our goal was to collect continuous samples of ice extending from the end of the last glacial episode through the early Holocene, including the

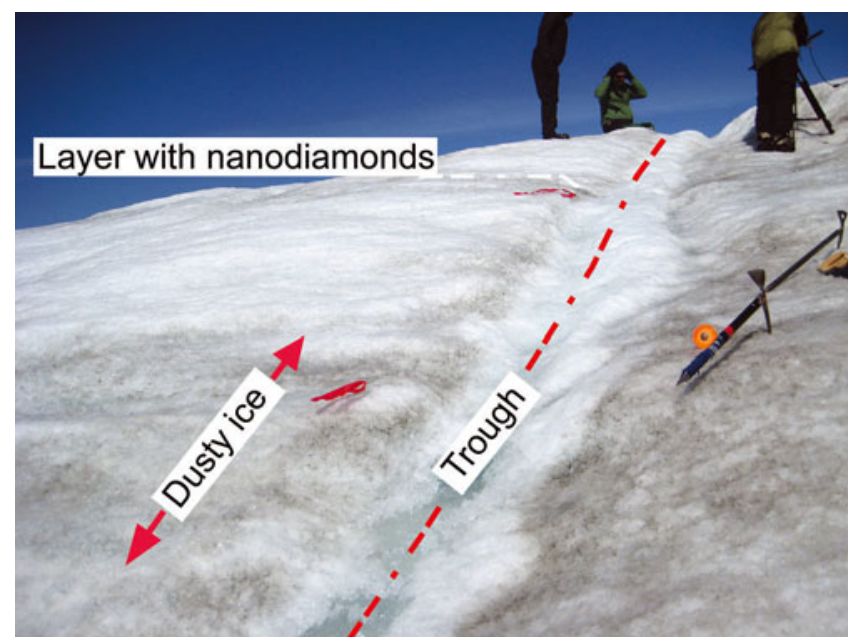

Fig. 2. Lower section of the trench at Kangerlussuaq, showing dusty ice of the last glacial episode, which contains very low abundances of NDs. This lower dusty section is immediately overlain by clearer, less dusty, whiter ice inferred to represent the Bølling-Allerød climatic episode, which contains few NDs. Position of layer containing abundant NDs is shown higher in the section.

1300 year long YD cooling episode. One of the authors (J.P.S.) has considerable experience sampling along the Greenland ice-sheet margin, and he identified a candidate for the YD-age section based on visual inspection of dust stratigraphy (Reeh and others, 2002; Petrenko and others, 2006). Our sampling was guided by the presence of clear, dust-poor ice of assumed early Holocene age, stratigraphically higher than a sharp visual change into dusty ice of inferred YD age, beneath which was another sharp change into clear ice of assumed Bølling-Allerød (BA) age that was preceded by dusty ice of assumed Late-glacial age (Figs 2 and 3). The dusty last-glacial ice was in turn separated by a major stratigraphic break from underlying clear ice that may date to the Eemian (the previous interglacial). The transitions between these climatic episodes are consistent with that

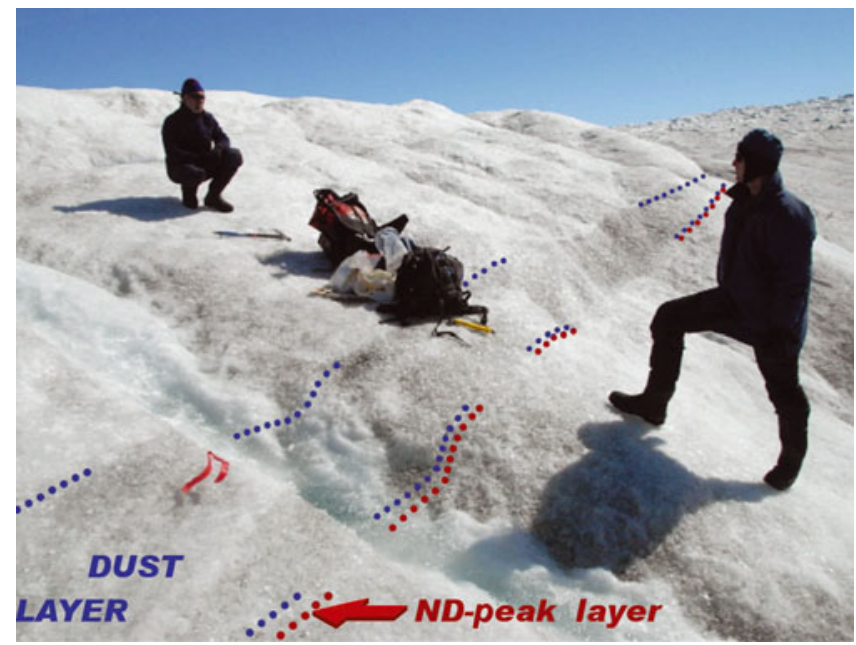

Fig. 3. Upper section of the trench at Kangerlussuaq showing a dusty ice layer sandwiched between clearer ice. The ND-peak layer lies immediately below the base of the dusty layer, as marked. Preliminary data suggest that the dusty layer may represent the YD cooling episode. If so, then the ND peak immediately pre-dates the base of the dusty layer marking the YD onset. 
expected for a Late-glacial through early Holocene ice sequence in Greenland.

To acquire surface samples, we scraped away $\sim 1 \mathrm{~cm}$ from the ice surface and then continuously collected at $15 \mathrm{~cm}$ intervals along the selected transect for $4.5 \mathrm{~m}$. For additional samples, we shifted $1 \mathrm{~m}$ parallel to the surface sample transect and excavated a $17 \mathrm{~m}$ long subsurface trench that included a deglacial age sequence extending from before the BA into the early Holocene. If the stratigraphic interpretation we have adopted is correct, the continuous sequence of ice samples we collected represents $\sim 6000$ years of ice, spanning the YD at a resolution of about every 50-100 years.

After returning to the laboratory, we measured stable oxygen isotope values in the melted water from these samples using standard analytical procedures. In addition, we searched for extraterrestrial material within the samples and identified the NDs and their allotropes using highresolution electron microscopy (HREM), transmission electron microscopy (TEM) and scanning transmission electron microscopy (STEM) to produce high-resolution lattice images and selected area electron diffraction (SAED) patterns, which display crystal lattice spacings that are uniquely attributable to the individual diamond polymorphs. (See Appendix for detailed methodology.)

Raman spectra were acquired using a Thermo Fisher Scientific DXR Raman Microscope by analyzing acidresistant residue on copper TEM grids with silicon monoxide film. Using the $532 \mathrm{~nm}$ laser, the beam size was $0.7 \mu \mathrm{m}$ with a resolution of $\pm 2.0 \mathrm{~cm}^{-1}$ at very low wattage $(0.5 \mathrm{~mW})$ to avoid damaging the diamonds. A $100 \times$ objective was used with a $25 \mu \mathrm{m}$ pinhole aperture.

\section{RESULTS AND DISCUSSION \\ Oxygen isotopes and stratigraphy}

Ice near the margins of the ice sheet at Kangerlussuaq was transported from its original accumulation area at higher icesheet elevations up to hundreds of kilometers from the east. Due to deformation and shearing, it was thinner when it reached the ice margin, causing the YD ice to vary unevenly in thickness. For example, at GISP2 the YD ice is $\sim 98 \mathrm{~m}$ thick (Meese and others, 1997), while away from central Greenland, at six sampling sites (Camp Century, Dye-3, Isunguata, Næsset, Paakitsoq and Kangerlussuaq) the YD thickness ranges from 1 to $50 \mathrm{~m}$, with an average of $12.8 \mathrm{~m}$. Near Kangerlussuaq, the inferred YD layer is $1 \mathrm{~m}$ thick, just

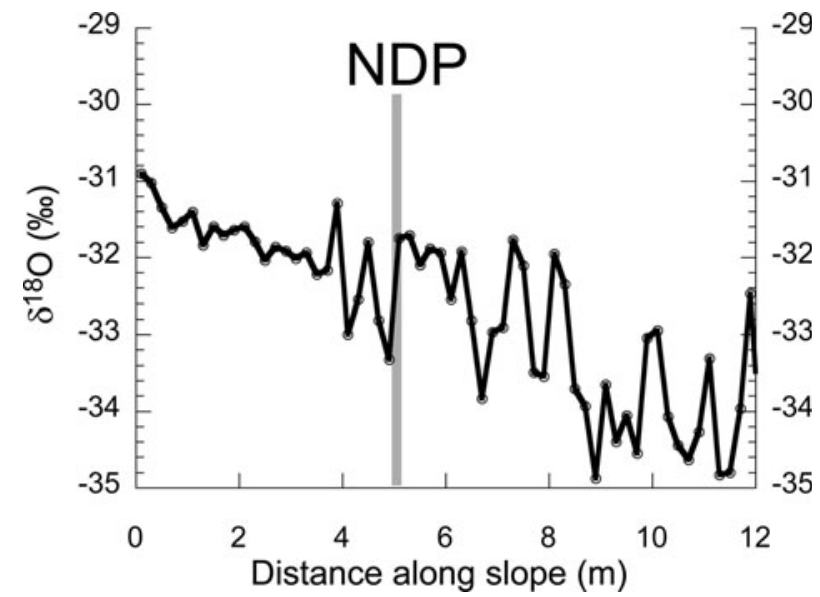

Fig. 4. Time series of $\delta^{18} \mathrm{O}$ values from the surface samples at the Kangerlussuaq site. The ND-peak layer is designated by a vertical gray line.

as it is along the ice margin at Paakitsoq, $\sim 350 \mathrm{~km}$ to the north (Table 1). Even though it is possible that a complete stratigraphic record has not been preserved at all locations along Greenland's margin, the ice profile studied at Kangerlussuaq displayed dust stratigraphy and isotopic values similar to deep ice-core records (Reeh and others, 2002; Petrenko and others, 2006), suggesting that the YD layer is in the correct stratigraphic position at that site.

We used the changes in $\delta^{18} \mathrm{O}$ values to constrain the age of the sequence through comparison with isotopic sequences described elsewhere in the Greenland ice sheet, including ice cores from Dye-3, Camp Century (Dansgaard and others, 1982) and GISP2 (Stuiver and Grootes, 2000). Our trench and surface samples, taken at relatively low resolution, yielded $\delta^{18} \mathrm{O}$ profiles for both trench and surface transects that exhibited a high degree of variability, but they still generally correspond to the distinctive sequence of warming/cooling episodes between the late last-glacial episode and early Holocene as recognized in the deep ice cores (Table 1). The observed $\delta^{18} \mathrm{O}$ values measured in trench and surface samples are about $-34.9 \%$ in the lower part of the profile (Fig. 4), and this is within the range of values for glacial ice reported from six proximal Greenland sites $\left(-33.0 \%\right.$ to $-42.0 \%$ ). Also, the $\delta^{18} \mathrm{O}$ values for the glacial episode differ by only $0.7 \%$ from Dye-3, the closest ice core (Dansgaard and others, 1982) located upstream from the Kangerlussuaq site. Significantly, since the Dye-3

Table 1. Characteristics of the Kangerlussuaq section compared to other sites reported for Greenland

\begin{tabular}{|c|c|c|c|c|c|c|c|c|c|}
\hline \multirow[t]{3}{*}{ Site } & \multicolumn{2}{|c|}{ Location } & \multirow[t]{2}{*}{ Elevation } & \multirow[t]{2}{*}{ Distance } & \multirow[t]{2}{*}{ YD } & \multicolumn{3}{|c|}{$\delta^{18} \mathrm{O}$ value } & \multirow[t]{3}{*}{ Source } \\
\hline & $\mathrm{N}$ & W & & & & Glacial & YD & Holocene & \\
\hline & & & $\mathrm{m}$ & $\mathrm{km}$ & $\mathrm{m}$ & $\%$ & $\%$ & $\%$ & \\
\hline Isunguata & 67.1617 & 50.16 & 313 & 5.9 & 12 & -33.9 & -31.0 & -28.0 & Reeh and others (2002) \\
\hline Paakitsoq & 69.4317 & 50.2667 & 350 & 250 & 1 & -39.6 & -39.0 & -32.5 & Reeh and others (2002) \\
\hline Kangerlussuaq & 67.1564 & 50.0233 & 366 & 0 & 1 & -34.9 & -33.3 & -31.0 & This paper \\
\hline Næsset & 67.1533 & 50.025 & 367 & 330 & 50 & -33.0 & -31.9 & -28.0 & Reeh and others (2002) \\
\hline Camp Century & 77.166 & 61.133 & 1120 & 1170 & 9 & -42.0 & -39.0 & -29.0 & Dansgaard and others (1982) \\
\hline Dye-3 & 65.1833 & 43.8167 & 2470 & 350 & 4 & -35.6 & -35.1 & -27.0 & Dansgaard and others (1982) \\
\hline GISP2 & 72.5883 & 38.4575 & 3183 & 750 & 98 & -43.6 & -40.1 & -29.4 & Stuiver and Grootes (2000) \\
\hline
\end{tabular}




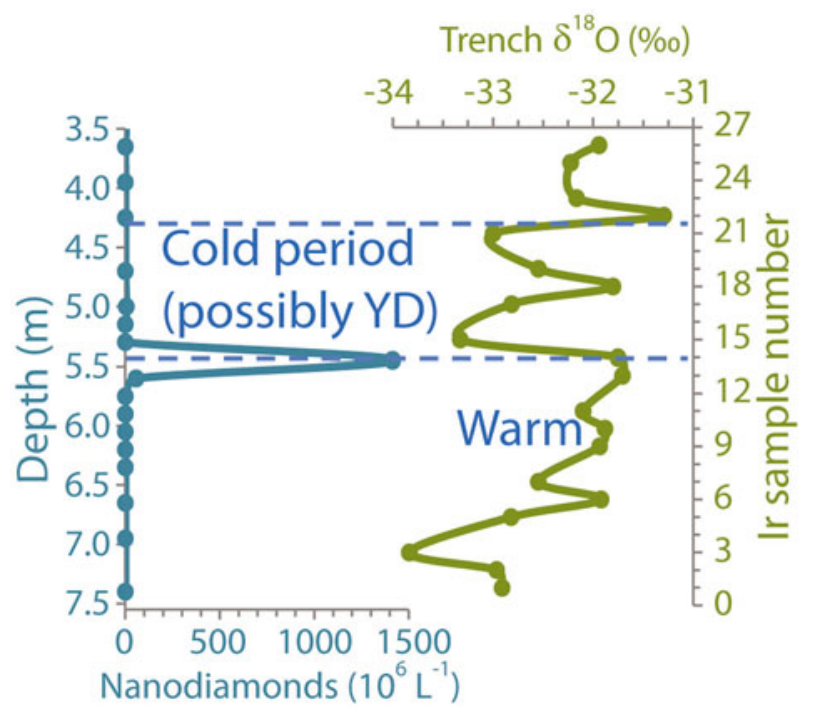

Fig. 5. Vertical abundance distribution of NDs from Table 2 compared with a low-resolution $\delta^{18} \mathrm{O}$ trench record that exhibits an apparent episode of cooling that may represent the YD. Distance down trench is shown at left and sample numbers are shown at right.

accumulation area is similar to Kangerlussuaq, that site is the most likely to reveal a comparable $\delta^{18} \mathrm{O}$ record (Table 1 ). Maximum $\delta^{18} \mathrm{O}$ values of about $-31.0 \%$ in the upper part of the Kangerlussuaq profile are also similar to the earliest Holocene values of about $-31.0 \%$ in Dye-3 (Dansgaard and others, 1982). The absence of higher values at Kangerlussuaq suggests that the younger part of the Holocene is absent and that the sampled profile does not extend further into the early Holocene, where values are typically about $-27.0 \%$ (Dansgaard and others, 1982, 1989; Reeh and others, 2002).

Based upon the dust stratigraphy and the $\delta^{18} \mathrm{O}$ values, the sequence at Kangerlussuaq is therefore consistent with a chronology that extends across the interval of deglaciation, ranging from the Late-glacial through the earliest Holocene. This deglacial sequence contains an interval of dustier ice with $\delta^{18} \mathrm{O}$ values (about $-33.0 \%$ ) that appear to represent the cooler conditions of the YD. It is noteworthy that the glacial $\delta^{18} \mathrm{O}$ values of the lower dusty ice contrast with the much higher $\delta^{18} \mathrm{O}$ values of the basal ice that appears to correlate with the Eemian episode (penultimate interglacial).

\section{Nanodiamonds}

Several authors report abundant NDs (n-diamonds, lonsdaleite, cubics and other allotropes) in the YDB layer dating to $12.9 \pm 0.1 \mathrm{ka}$ at seven terrestrial sites across North America (Kennett and others, 2009a,b). Now, in an ice layer from the Kangerlussuaq study site, we have detected a ND-rich peak in a layer of ice (ND-peak layer) that is estimated to contain total ND abundances of 5-50 parts per billion (ppb), equivalent to $1-10 \times 10^{9}$ per $\mathrm{cm}$ of ice (Fig. 5; Table 2). These values are consistent with the lower end of the range of 10-3700 ppb estimated at terrestrial North American YDB sites (Kennett and others, 2009a). Analytical results from this pilot study for lonsdaleite and n-diamonds are provided in Figures 6 and 7 and Table 3.

Researchers have produced lonsdaleite experimentally at high temperatures $\left(1000-1700^{\circ} \mathrm{C}\right)$ with pressures of $>15 \mathrm{GPa}$ $\left(2 \times 10^{6} \mathrm{psi}\right)$, followed by rapid quenching (DeCarli and
Table 2. Estimated concentrations of NDs by depth and sample for the Kangerlussuaq section. Based on HREM images, approximately one-third of the total NDs are estimated to be lonsdaleite, one-third are n-diamonds and the balance is comprised of other diamond allotropes

\begin{tabular}{lrr}
\hline Depth & Sample No. & \multicolumn{2}{c}{ NDs } \\
$\mathrm{m}$ & & $\mathrm{mL}^{-1}$ \\
\hline 3.15 & 29 & 0 \\
3.60 & 26 & 6790 \\
3.90 & 24 & 0 \\
4.20 & 22 & 0 \\
4.65 & 19 & 0 \\
4.95 & 17 & 537 \\
5.10 & 16 & 5291 \\
5.25 & 15 & 521 \\
5.40 & 14 & 1414730235 \\
5.55 & 13 & 124 \\
5.70 & 12 & 689 \\
5.85 & 11 & 1127 \\
6.00 & 10 & 630 \\
6.15 & 9 & 1167 \\
6.30 & 8 & 0 \\
6.60 & 6 & 591 \\
6.90 & 4 & 83 \\
7.35 & 1 & $1 \times 10^{9}$ \\
Total: & & \\
& &
\end{tabular}

others, 2002), just as would occur during a hypervelocity cosmic impact. In addition, lonsdaleite has been experimentally produced by carbon-vapor deposition (CVD), whereby an oxygen-deficient (hypoxic), carbon-rich $13000^{\circ} \mathrm{C}$ vapor was quenched onto a warm substrate $\left(800-1200^{\circ} \mathrm{C}\right)$ at typical atmospheric pressure (Maruyama and others, 1992), conditions which could occur in an impact fireball. Neither of these sets of experimental

Table 3. Lattice d-spacings for n-diamonds and lonsdaleite. The second column for each type shows accepted values for each particular lattice, and the third column displays the observed lattice $\mathrm{d}$-spacings from the Greenland samples, indicating good matches with each calculated spacing. In crystallography, Miller indices, expressed as hkl values, are a notational system used to describe the orientation of a family of planes of atoms as revealed by exposure to X-rays or a beam of electrons. A 'd-spacing' value is the spacing (herein as $\mathrm{nm}$ ) between adjacent planes. Any crystal, such as diamond, displays a uniquely individual set of $d$-spacings (Wenk and Bulakh, 2004)

\begin{tabular}{cccccc}
\hline \multicolumn{3}{c}{ N-diamonds } & \multicolumn{3}{c}{ Lonsdaleite } \\
$\mathrm{hkl}$ & $d$ (calc) & $d$ (obs) & $\mathrm{hkl}$ & $d$ (calc) & $d(\mathrm{obs})$ \\
& $\mathrm{nm}$ & $\mathrm{nm}$ & & $\mathrm{nm}$ & $\mathrm{nm}$ \\
& & & & & \\
\hline 111 & 0.206 & $0.205-0.207$ & 100 & 0.218 & $0.218-0.220$ \\
200 & 0.178 & $0.176-0.180$ & 002 & 0.206 & $0.206-0.207$ \\
220 & 0.126 & $0.124-0.127$ & 101 & 0.193 & $0.190-0.193$ \\
311 & 0.107 & $0.106-0.108$ & 102 & 0.150 & $0.150-0.154$ \\
222 & 0.104 & $0.102-0.105$ & 110 & 0.126 & $0.125-0.126$ \\
400 & 0.090 & $0.088-0.092$ & 103 & 0.116 & $0.117-0.118$ \\
& & & 200 & 0.109 & $0.108-0.109$
\end{tabular}



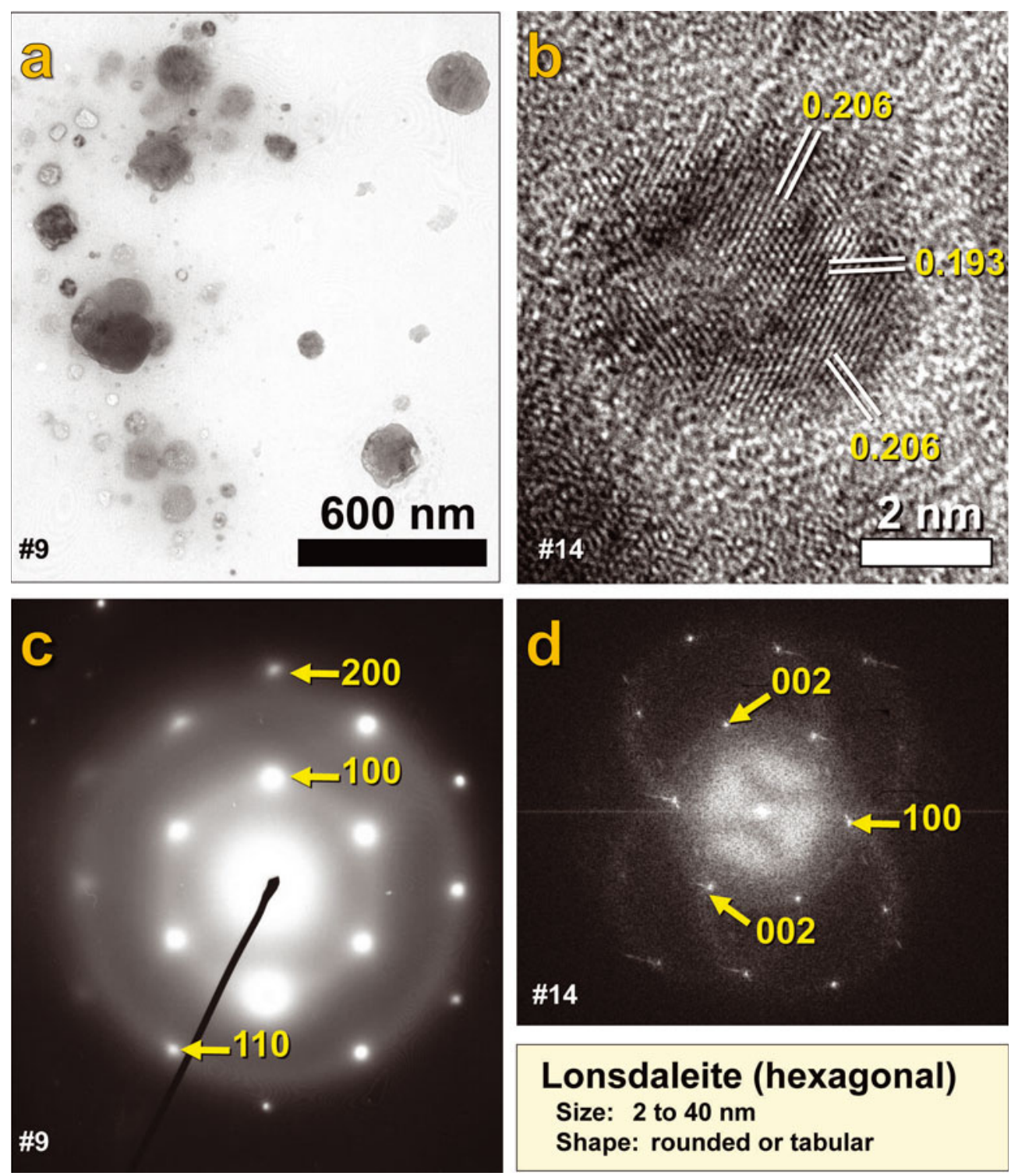

\section{Lonsdaleite (hexagonal)}

Size: 2 to $40 \mathrm{~nm}$

Shape: rounded or tabular

Fig. 6. Lonsdaleite analyses. (a) TEM image of group of lonsdaleite crystals. (b) HREM image showing characteristic lonsdaleite lattice spacings. (c) SAED pattern of lonsdaleite. (d) Fast Fourier transform (FFT) of lonsdaleite ND crystal. All values (Miller indices) are consistent with each other and with the published lattice spacings for lonsdaleite as shown in Table 3. Sample numbers, as referenced in Table 2, are shown in the lower left of each image.

conditions is known to occur in nature, supporting the observation that lonsdaleite has never been confirmed in association with terrestrial diamonds (DeCarli and others, 2002). Lonsdaleite has only previously been found on Earth either inside fallen meteorites (Hanneman and others, 1967), in which they formed by shock metamorphism in space, or in association with terrestrial impact craters (DeCarli and others, 2002), in which they formed by shock metamorphism during hypervelocity impact. Consequently, the presence of lonsdaleite is considered a robust indicator of the influx of cosmic material or of a cosmic impact, since there are no known natural terrestrial processes, such as tectonism, volcanism or biomass burning, that are accepted as being capable of providing the requisite conditions for lonsdaleite formation (DeCarli and others, 2002).

Of the various polytypes in the ND-peak layer, lonsdaleite accounts for approximately one-third of the total NDs detected in the ND-rich layer. These are typically monocrystalline, displaying a generally rounded morphology with slightly irregular edges (Fig. 6). Lonsdaleite varies from $\sim 2$ to $40 \mathrm{~nm}$ in diameter, with most crystals falling at the lower end of that range; they are crystallographically identical to lonsdaleite from impact craters, such as Popigai, Russia (Koeberl and others, 1997), except that Popigai diamonds are angular or tabular rather than rounded. We detected no lonsdaleite in ice younger than the ND-peak layer, but low concentrations of $<0.003$ parts per trillion (ppt) were detected in older adjacent layers, possibly due to ice ablation and downslope transport of material from the ND-peak layer.

$\mathrm{N}$-diamonds account for approximately an additional one-third of the total NDs detected in the ND-peak layer. Analytical results from HREM, TEM, electron energy-loss spectroscopy (EELS) and Raman are shown for n-diamonds in Figures 7-9 and Table 3. Energy-dispersive X-ray spectroscopy (EDS) confirms that the n-diamonds are composed of elemental carbon $(\sim 99 \%)$, and EELS indicates that the crystalline habit of selected crystals matches that of $\mathrm{n}$-diamond (Fig. 8), displaying a mix of $\mathrm{sp}^{2}$ and $\mathrm{sp}^{3}$ bonding (Konyashin and others, 2001; Peng and others, 2001). In the 

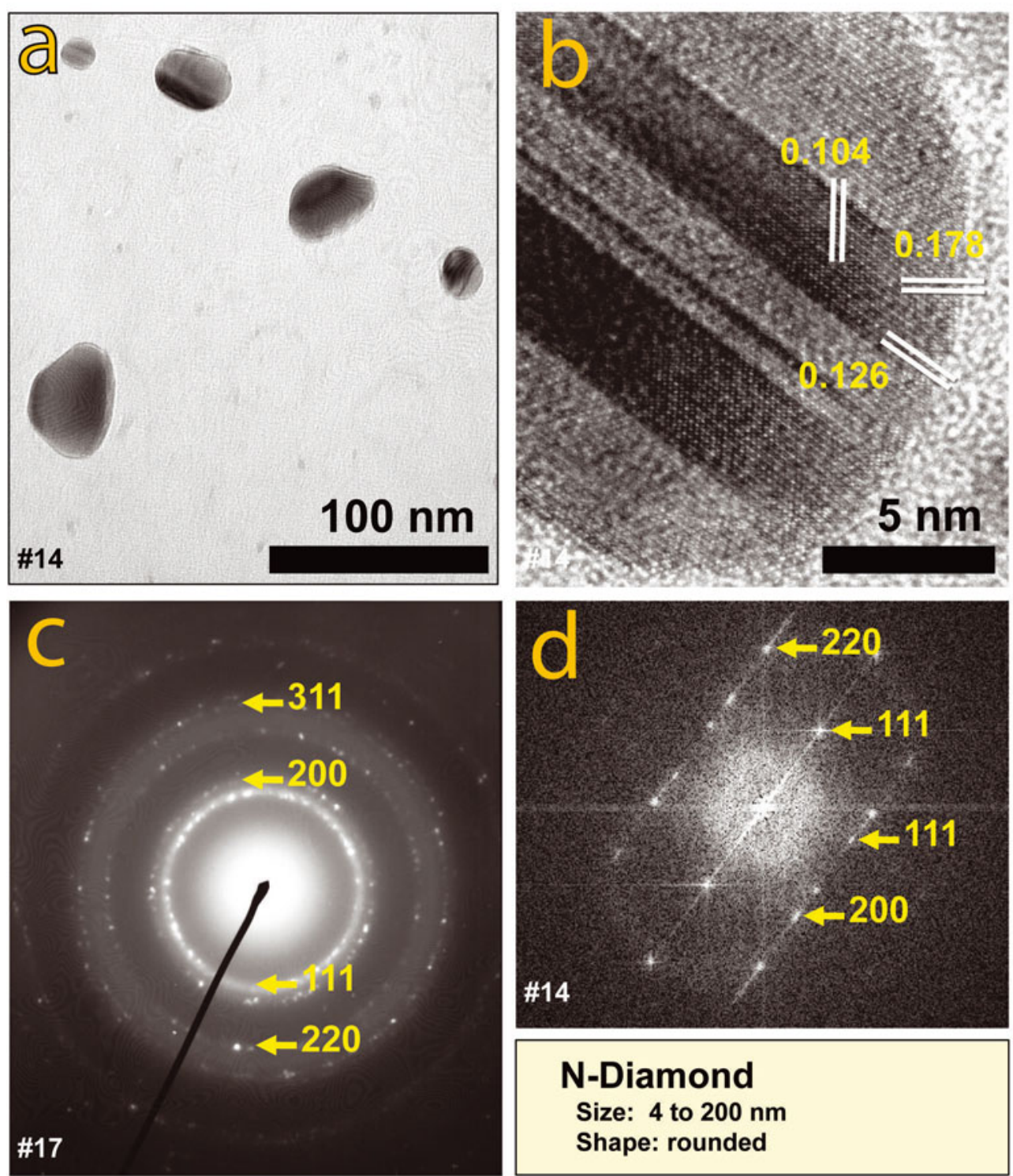

\section{N-Diamond \\ Size: 4 to $200 \mathrm{~nm}$ \\ Shape: rounded}

Fig. 7. N-diamond analyses. (a) TEM image of group of n-diamonds. (b) HREM image showing characteristic n-diamond lattice spacings. Dark and light alternating lattice bands indicate twinned crystals, a common characteristic of n-diamonds in the Greenland section. In contrast, lonsdaleite from Greenland (Fig. 6) displays no indication of twinning. (c) SAED pattern of n-diamonds. (d) FFT of n-diamond crystal. All lattice spacings (Miller indices) are consistent with each other and with published spacings for $\mathrm{n}$-diamonds as shown in Table 3. Sample numbers, as referenced in Table 2, are shown in the lower left of each image.

laboratory, $\mathrm{n}$-diamonds can be produced experimentally by oxygen-deficient (hypoxic) TNT detonation (Yamada and Sawaoka, 1994) and are typically rounded and often polycrystalline, ranging from $\sim 2$ to $40 \mathrm{~nm}$ (fig. 2.9 by T.L. Daulton in Shenderova and Gruen, 2007). For n-diamonds from Greenland's ND-peak layer, HREM and TEM imaging reveal that they are also typically rounded and often polycrystalline, ranging in size from 4 to $200 \mathrm{~nm}$, with most falling within the range $4-10 \mathrm{~nm}$. Therefore, the shape and size of the Greenland n-diamonds suggest that they formed not by shock metamorphism but rather by processes such as high-temperature CVD and/or high-explosive detonation, which duplicate conditions known to occur during a cosmic impact.

Our analysis of the Greenland sequence revealed low background concentrations of NDs in 16 non-ND-peak layer ice samples spanning an estimated interval of $\sim 6000$ years (Table 2). We detected no lonsdaleite within younger ice above the ND-peak layer, but we found lonsdaleite and n-diamonds at low concentrations intermittently in older ice below the ND-peak layer. The presence of these apparently older NDs most likely results from redeposition by ice ablation of NDs found in the peak layer. For the entire ice transect analyzed, spanning an estimated interval from $\sim 16$ to $\sim 11 \mathrm{ka}$, only one ND peak was discovered, and that occurs in clear ice immediately preceding a dusty ice layer that is consistent with the onset of the YD cooling episode. Other than that, the background levels of NDs are about the same whether found in clear ice or dusty ice, indicating that there is no apparent direct relationship between the abundances of terrestrial dust and NDs. The average background of non-peak-layer NDs in the $\sim 6000$ years of sampled intervals in this Greenland section ranges from 0.0 to $0.1 \mathrm{ppt}$ and averages $0.01 \mathrm{ppt}$, which is lower than in the ND-peak layer by a factor of 500000-5000000 times (Table 2). 

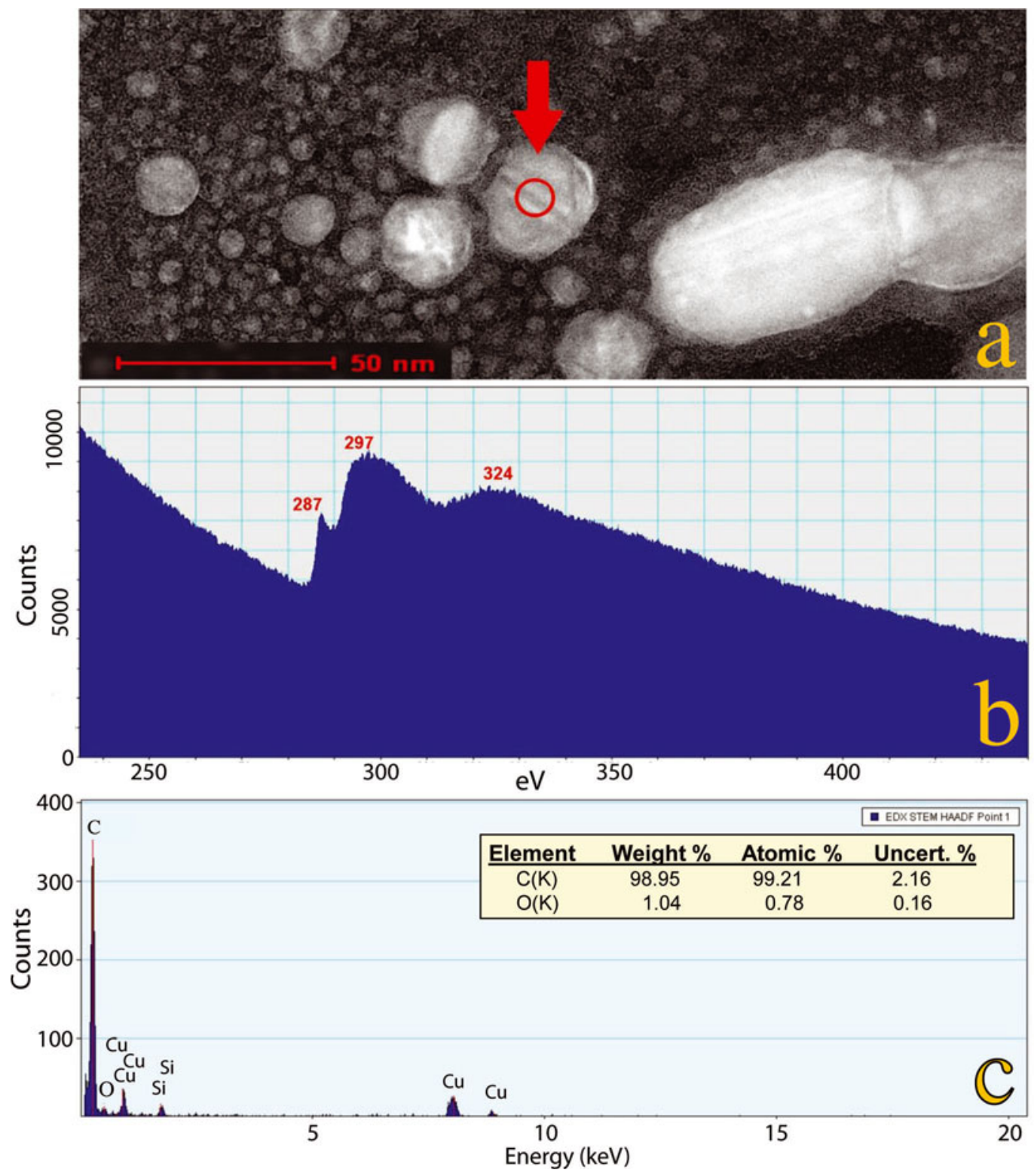

Fig. 8. Energy-dispersive X-ray spectroscopy (EDS) and electron energy-loss spectroscopy (EELS) analyses of an n-diamond from the ND-peak layer in the Greenland section (sample No. 14; 5.40 m). (a) A dark-field STEM image of many n-diamonds, one of which (arrow) displays diagonal bands that are characteristic of twinning, a common condition for the Greenland n-diamonds. Several analyses were conducted in the area of the spot circled, as follows: (b) An EELS core-loss spectrum revealed a pre-peak at $287 \mathrm{eV}$ and a sigma peak at $297 \mathrm{eV}$, both of which are characteristic of n-diamonds. A low-loss spectrum (not shown) displayed a peak at $22 \mathrm{eV}$, and this in addition to the previous coreloss eV values matches those reported for $\mathrm{n}$-diamonds (Konyashin and others, 2001; Peng and others, 2001). The n-diamond values differ distinctively from the respective values for other carbon allotropes, such as cubic diamonds, lonsdaleite, graphite and amorphous carbon (Peng and others, 2001). (c) EDS analysis confirming that the crystal analyzed is dominantly elemental carbon ( 99\%). Corrections were made for small amounts of copper $(\mathrm{Cu})$ from the TEM grid and silicon (Si) from the EDS detector.

\section{Alternate hypotheses}

Regarding the origin of the Greenland ND-peak layer, there are several hypotheses, one of which is that the lonsdaleite and n-diamonds have not been conclusively identified. Obviously, it is important that care be taken to correctly identify the ND allotropes and differentiate them from other mineral species, which can appear similar. For example, lonsdaleite and $\mathrm{n}$-diamonds display diffraction lines with values within a few percent of the lines of other minerals such as quartz, rutile, copper, graphite and graphene. However, when occasionally encountering those crystals during TEM analysis, we found their shapes to be morphologically unlike the spherical to highly rounded Greenland diamonds. Furthermore, all of the alternate crystals display additional diffraction lines and/or are missing some lines (e.g. quartz displays additional non-diamond lines at 0.426, 0.334 and $0.246 \mathrm{~nm}$; graphite displays an additional line at $0.335 \mathrm{~nm}$; and graphene is missing the lonsdaleite lines of 0.193 and $0.150 \mathrm{~nm}$ ), making it relatively straightforward to differentiate those crystals from diamonds. Furthermore, SAED, EDS, EELS and Raman analyses all clearly indicate that the reported Greenland diamonds are dominantly carbon ( 90-99\%), while quartz, rutile and copper are barely detectable $(\sim 0 \%$ to $\sim 2 \%)$, thus eliminating the possibility that the dominant minerals are other than diamonds. In fact, the combination of these various analyses is considered to provide conclusive identification of any unknown crystal. Thus, multiple lines of evidence indicate 
that the Greenland ND-peak layer contains highly abundant lonsdaleite and n-diamonds, which are morphologically and analytically indistinguishable from the lonsdaleite and n-diamonds found across North America in YDB carbon spherules and sediments.

A second hypothesis is that the NDs resulted from an unusually intense, non-catastrophic influx of ET diamonds, such as from a heavy meteor shower. To test this hypothesis, we surveyed the literature for ET diamond morphologies, and found that many researchers have analyzed meteoritic diamonds and presolar diamonds in cosmic dust, and reported finding angular, euhedral and/or plate-like NDs (e.g. Grady and others, 1995; Shenderova and Gruen, 2007; Marosits and others, 2008). In addition, T.L. Daulton reported finding quasi-rounded, decahedral, multiply twinned cubic NDs (fig. 2.11 by Daulton in Shenderova and Gruen, 2007 but, to our knowledge, no one has reported finding rounded monocrystalline NDs within cosmic material. In addition, we prepared our own diamond extracts from three meteorites and confirmed the presence of lonsdaleite in a ureilite (NWA 2971) and cubic diamonds in two carbonaceous chondrites (Allende and Murchison) with the same angular or euhedral morphology as reported in other meteorites, but those NDs are dissimilar in morphology to the Greenland lonsdaleite and n-diamonds. Testing continues, but so far we have detected no n-diamonds in any meteoritic samples, nor, to our knowledge, have other researchers conclusively identified n-diamonds, rounded or otherwise, in any meteoritic samples. Since the rounded n-diamonds found in Greenland are unlike any diamonds found in meteorites or presolar grains, this difference strongly suggests that they did not arrive from space. Instead, it appears that they formed upon Earth impact, most likely through a process such as hightemperature CVD and/or high-explosive detonation, which typically produces rounded laboratory NDs that are identical to those from Greenland (fig. 2.9 by Daulton in Shenderova and Gruen, 2007). If the Greenland NDs did form during an Earth impact, then carbonaceous material in terrestrial target rocks, biomass and/or possibly the impactor supplied their carbon.

Another hypothesis is that the Greenland ND-peak abundances may simply have resulted from a small localized meteorite impact and that the diamond-rich YDB layer in North America is not the same as the Greenland ND-peak layer. However, this seems unlikely since the Greenland NDpeak layer appears to have been deposited between 14.6 and $11.6 \mathrm{ka}$, and is the only peak layer apparent in that interval of ice. In all the sample profiles studied in Greenland, throughout North America and across Europe, an area spanning $\sim 12000 \mathrm{~km}$ and distributed across $\sim 10 \%$ of the Earth, there is only one ND peak at $\sim 12.9 \pm 0.1 \mathrm{ka}$, which is evident in each of the sedimentary sequences that span many thousands of years. Therefore, the simplest and most compelling explanation is that the ND-peak layer in Greenland is the same as the ND-rich continental YDB.

The final, and preferred, hypothesis is that the Greenland ND-peak layer, which appears to date to the onset of the YD, is the result of a major impact event. In support of this, the continent-wide Cretaceous-Tertiary $(\mathrm{K}-\mathrm{T})$ boundary layer is accepted to contain NDs that did not arrive from space inside the impactor, but rather crystallized from carbon-rich terrestrial material during that hypervelocity impact (Gilmour and others, 1992). During our Greenland research, we also examined NDs extracted from $\mathrm{K}-\mathrm{T}$ sediments collected

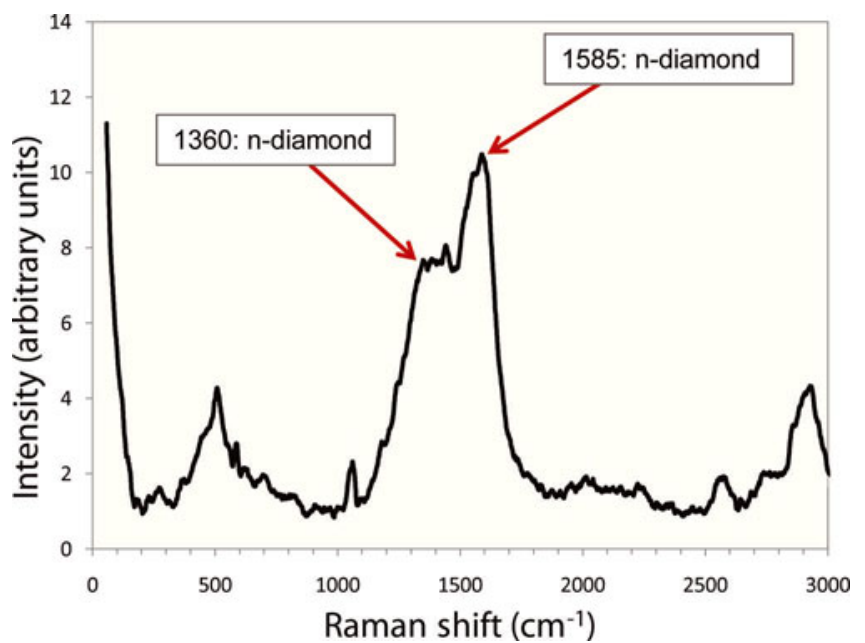

Fig. 9. Acid-resistant residue from the Greenland ND-peak layer (No. 14) analyzed by Raman spectroscopy reveals several broad peaks. These peaks are centered at 1360 and $1585 \mathrm{~cm}^{-1}$, which are characteristic values for n-diamond (Konyashin and others, 2006); the $1585 \mathrm{~cm}^{-1}$ peak is the largest apparent in this spectrum. There are no visible peaks for rutile or copper, eliminating the possibility that $\mathrm{n}$-diamonds are those minerals, all of which have similar, though distinctive, SAED patterns. The Raman spectra, along with SAED patterns and EDS, reveal the likely presence of abundant amorphous carbon intermixed with the n-diamonds. Lonsdaleite is not readily apparent in this Raman spectrum, possibly due to its much lower abundance relative to $\mathrm{n}$-diamonds.

in Caravaca, Spain, and Needles Point, New Zealand. The impact layers at both sites display substantial abundances of lonsdaleite and n-diamonds having the same morphology and range of sizes as those found in the Greenland samples (Bunch and others, 2008; West and others, 2009), while background levels were at zero or very low, as they are in Greenland. Likewise, NDs extracted from YDB samples collected in North America and Europe display the same rounded morphology and range of sizes as those found in the $\mathrm{K}-\mathrm{T}$ sediments and in Greenland (Kennett and others, 2009a). ND abundances from the YDB are found to range up to $3700 \mathrm{ppb}$, which is lower than, but comparable to, the lower values for the $\mathrm{K}-\mathrm{T}$ impact event, which has ND abundances from $\sim 3200$ to $45000 \mathrm{ppb}$. Similarly, Greenland ND abundances are found at up to $5 \times 10^{6}$ times above background and are comparable to YDB abundances of NDs at other sites. Thus, since the type and morphology of the Greenland NDs indicate that they are not meteoritic or presolar diamonds, the presence of the ND-peak layer in Greenland is most consistent with a major impact event, such as occurred at the $\mathrm{K}-\mathrm{T}$ boundary.

Of course, it is possible that the NDs in Greenland formed through some as-yet undiscovered natural process other than cosmic impact; however, that seems unlikely, since intense diamond research spanning more than a century indicates that the formation of NDs, and lonsdaleite in particular, requires extraordinary temperature, pressure and redox conditions that rule out natural processes that occur either on or below the surface of the Earth (DeCarli and others, 2002). Thus, since the YDB is the only other known continent-wide diamond layer in the entire tested geologic record other than that at the $\mathrm{K}-\mathrm{T}$ boundary, the most consistent hypothesis is that NDs in the YDB formed during a major cosmic impact event. 


\section{Future work}

The data presented herein result from a preliminary study. The discovery of a potential postglacial peak in NDs in the Greenland ice sheet is exciting, and although a few unanswered questions have arisen, we are presenting our results to stimulate further investigation and debate. For example, this study was undertaken using Greenland ice samples taken at relatively coarse resolution, and, to significantly refine the findings presented here, higherresolution sampling is needed. Further areas of potential exploration should include: (1) more precise dating through use of $\delta^{18} \mathrm{O}$; (2) more detailed geochemical time-series measurements, including for osmium and iridium; (3) utilization of improved diamond-extraction procedures to minimize acid-resistant amorphous carbon, which currently limits available analytical procedures; and (4) expanded analyses of diamond allotropes, such as by Raman spectroscopy. All of these analyses require more extensive, higherresolution sampling in Greenland.

\section{CONCLUSIONS}

The discovery in Greenland ice of abundant n-diamonds and lonsdaleite, along with other possible diamond allotropes, represents the only known depositional layer of free diamonds yet reported from glacial ice anywhere on Earth (e.g. ice sheets and caps; alpine glaciers). An inferred last deglacial age for the ND-peak layer is based on dust stratigraphy and constrained by oxygen isotopic values of the ice. This stratigraphy suggests that the ND-peak layer was deposited after the end of the last glacial episode (14.6 ka) and prior to the earliest Holocene $(11.6 \mathrm{ka})$, which is consistent with the date of formation of the YDB in North America at $12.9 \pm 0.1 \mathrm{ka}$. Also, the ND-peak layer occurs immediately below the base of an especially dusty ice zone, which, according to the dust stratigraphy, may represent the onset of the YD cooling episode. However, higher-resolution stratigraphic studies are needed to confirm this age.

Since lonsdaleite is only known to form during hightemperature/high-pressure cosmic events, its presence currently can be explained in only two ways: either the lonsdaleite arrived already formed within meteoritic material or it crystallized during a hypervelocity impact with the Earth's surface. The rounded morphology, range of allotropes and very high abundance of NDs in the ND-peak layer appear to exclude the first possibility, arrival inside meteoritic material. Regarding a possible impact event, the Greenland NDs are identical to those found in the $\mathrm{K}-\mathrm{T}$ impact layers, so the presence of rounded n-diamonds and lonsdaleite in Greenland ice suggests that a large cosmic impact occurred, and further investigations are necessary to determine the nature and scope of this event. In summary, since continent-wide layers containing NDs are only known to exist at the K-T boundary and the YDB, then the existence of this layer in Greenland ice appears consistent with the occurrence of a major impact event that correlates with the nanodiamond-rich YDB in North America at $12.9 \pm 0.1 \mathrm{ka}$.

\section{ACKNOWLEDGEMENTS}

Research was partially funded by the US National Oceanic and Atmospheric Administration (Department of Commerce grant NA08OAR4310867) and the US National Science Foundation (grant ATM-0713769). HREM work was conducted at the CAMCOR High Resolution Facility in the Lorry I. Lokey Laboratories at University of Oregon with support from the Office of Research. We thank the FEI Company for their generous support and technical assistance with HREM (FEI Titan ${ }^{\text {TM }}$ 80-300 scanning/transmission electron microscope). We thank Thermo Fisher Scientific, Inc., S. Heier, T. Deschaines and J. Hellgeth for their generous support and technical assistance with the Thermo Fisher Scientific DXR Raman Microscope. We also gratefully acknowledge PBS's NOVA series, E. Hadingham (senior science editor), D. Hamilton (producer-director) and S. Pyne (assistant producer) for encouragement and financial support for fieldwork in Greenland by A.V.K., P.A.M. and J.P.S. We thank the three reviewers for their valuable comments and suggestions.

\section{REFERENCES}

Bunch, T.E. and 9 others. 2008. Hexagonal diamonds (lonsdaleite) discovered in the K/T impact layer in Spain and New Zealand. [Abstr. PP13C-1476.] Eos, 89(53), Fall Meet. Suppl.

Dansgaard, W. and 6 others. 1982. A new Greenland deep ice core. Science, 218(4579), 1273-1277.

Dansgaard, W., J.W.C. White and S.J. Johnsen. 1989. The abrupt termination of the Younger Dryas climate event. Nature, 339(6225), 532-534.

DeCarli, P.S., E. Bowden, A.P. Jones and G.D. Price. 2002. Laboratory impact experiments versus natural impacts events. In Koeberl, C. and K.G. MacLeod, eds. Catastrophic events and mass extinctions: impacts and beyond. Boulder, CO, Geological Society of America, 595-605. (GSA Special Paper 356.)

Firestone, R.B. and 25 others. 2007. Evidence for an extraterrestrial impact 12,900 years ago that contributed to the megafaunal extinctions and the Younger Dryas cooling. Proc. Natl. Acad. SCi. USA (PNAS), 104(41), 16,016-16,021.

Gilmour, I., S.S. Russell, J.W. Arden, M.R. Lee, I.A. Franchi and C.T. Pillinger. 1992. Terrestrial carbon and nitrogen isotopic ratios from Cretaceous-Tertiary boundary nanodiamonds. Science, 258(5088), 1624-1626.

Grady, M.M., M.R. Lee, J.W. Arden and C.T. Pillinger. 1995. Multiple diamond components in Acfer 182. Earth Planet. Sci. Lett., 136(3-4), 677-692.

Hanneman, R.E., H.M. Strong and F.P. Bundy. 1967. Hexagonal diamonds in meteorites: implications. Science, 155(3765), 995-997.

Huss, G.R. and R.S. Lewis. 1995. Presolar diamond, SiC, and graphite in primitive chondrites: abundances as a function of meteorite class and petrologic type. Geochim. Cosmochim. Acta, 59(1), 115-160.

Kennett, D.J. and 8 others. 2009a. Nanodiamonds in the Younger Dryas boundary sediment layer. Science, 323(5910), 94.

Kennett, D.J. and 16 others. 2009b. Shock-synthesized hexagonal diamonds in Younger Dryas boundary sediments. Proc. Natl. Acad. Sci. USA (PNAS), 106(31), 12,623-12,628.

Koeberl, C., V.L. Masaitis, G.I. Shafransovsky, I. Gilmour, F. Langenhorst and M. Shrauder. 1997 Diamonds from the Popigai impact structure, Russia. Geology, 25(11), 967-970.

Konyashin, I. and 6 others. 2001. A new carbon modification: 'ndiamond' or face-centred cubic carbon? Diamond Rel. Mater., 10(1), 99-102.

Konyashin, I., V. Khvostov, V. Babaev, M. Guseva, J. Mayer and A. Sirenko. 2006. A new hard allotropic form of carbon: dream or reality? Int. J. Refract. Metal Hard Mater., 24(1-2), 17-23.

Marosits, E., T.L. Daulton, C. Sudek and U. Ott. 2008. What we do to meteoritic nanodiamonds when we heat them. In Proceedings of the 39th Lunar and Planetary Science Conference, 10-14 March 2008, League City, Texas, USA. Abstract 1252. Houston, TX, Lunar and Planetary Institute. CD-ROM. 
Maruyama, K., M. Makino, N. Kikukawa and M. Shiraishi. 1992. Synthesis of hexagonal diamond in a hydrogen plasma jet. J. Mater. Sci. Lett., 11(2), 116-118.

Meese, D.A. and 8 others. 1997. The Greenland Ice Sheet Project 2 depth-age scale: methods and results. J. Geophys. Res., 102(C12), 26,411-26,423.

Peng, J.L., L.A. Bursill, B. Jiang, J.O. Orwa and S. Prawer. 2001. Growth of c-diamond, n-diamond and i-carbon nanophases in carbon-ion-implanted fused quartz. Philos. Mag. B, 81(12), 2071-2987.

Petrenko, V.V., J.P. Severinghaus, E.J. Brook, N. Reeh and H. Schaefer. 2006. Gas records from the West Greenland ice margin covering the Last Glacial Termination: a horizontal ice core. Quat. Sci. Rev., 25(9-10), 865-875.

Reeh, N., H. Oerter and H.H. Thomsen. 2002. Comparison between Greenland ice-margin and ice-core oxygen-18 records. Ann. Glaciol., 35, 137-144.

Shenderova, O.A. and D.M. Gruen. 2007. Ultrananocrystalline diamond: synthesis, properties, and applications. Norwich, NY, William Andrew.

Stuiver, M. and P.M. Grootes. 2000. GISP2 oxygen isotope ratios. Quat. Res., 53(3), 277-284.

Wenk, H.R. and A. Bulakh. 2004. Minerals: their constitution and origin. Cambridge, etc., Cambridge University Press.

West, A., J. Kennett, D.J. Kennett, T.E. Bunch, T.W.J. Stafford and W.S. Wolbach. 2009. Are nanodiamonds evidence for a Younger Dryas impact event? [Abstr. PP23D-01.] Eos, 90(52), Fall Meet. Suppl.

Yamada, K. and A.B. Sawaoka. 1994. Very small spherical crystals of distorted diamond found in a detonation product of explosive/ graphite mixtures and their formation mechanism. Carbon, 32(4), 665-673.

Yates, P.D., J.W. Arden, I.P. Wright, C.T. Pillinger and R. Hutchison. 1992. A search for pre-solar material within an acid-resistant residue of Greenland cryoconite. Meteoritics, 27(3), 309.

\section{APPENDIX}

\section{Stable oxygen isotope $\delta^{18} \mathrm{O}$ measurements from glacial ice}

We utilized a Multiprep peripheral running both Sira software and a Prism-Up software package. We aliquoted $375 \mu \mathrm{L}$ of sample water to a Wheaton $1.0 \mathrm{~mL}$ V-Vial (Wheaton \#986284). After transferring a suite of samples to a glove bag and sealing the bag, the atmosphere was purged from the bag with a stream of $\mathrm{CO}_{2}$ gas. When $1 \mathrm{~atm}$ of $\mathrm{CO}_{2}$ was reached within the glove bag, screw tops were placed on each sample vial. These screw tops contain a penetrable septa backed by a Kel-F disc. The water samples were then equilibrated with $\mathrm{CO}_{2}$ overnight at $30^{\circ} \mathrm{C}$. When equilibrated the sample gas was admitted to the Multiprep peripheral via a needle that pierces both the septa and Kel-F disc, and dried by passing through a trap held at $-80^{\circ} \mathrm{C}$. The gas was then transferred into the dual inlet of the Sira mass spectrometer for measurement. We report the difference in absolute oxygen isotopic ratios between samples of Greenland ice and Vienna Standard Mean Ocean Water (V-SMOW). By measuring the difference in isotopic ratios, we can more precisely detect fluctuations or changes in values than if we only reported absolute isotopic values. Data are reported as enrichment, $\delta$, expressed as per mil (\%o) relative to V-SMOW (Stuiver and Grootes, 2000). A calibrated working standard gas of tank $\mathrm{CO}_{2}$ is used in the laboratory. Sample values are measured against this working standard gas, and then converted and reported relative to the international standard $\mathrm{V}$-SMOW by also measuring three working water standards on a daily
Table 4. Stable oxygen isotope standards

\begin{tabular}{|c|c|c|}
\hline Standard & Comments & Value \\
\hline V-SMOW & & 0.00 \\
\hline SLAP & & -55.5 \\
\hline GISP & & -24.8 \\
\hline IAEA OH-1 & & -0.1 \\
\hline IAEA OH-2 & & 3.3 \\
\hline IAEA OH-3 & & -8.7 \\
\hline IAEA OH-4 & & -15.3 \\
\hline BBB & Big Bear Brook water from Maine, USA & -8.5 \\
\hline ASS & Antarctic surface snow & -25.7 \\
\hline LAP & Light Antarctic precipitation & -40.3 \\
\hline
\end{tabular}

basis. These laboratory standards are reported in Table 4. The working standards are close in isotopic value to the international water standards V-SMOW and GISP and have been calibrated directly to these internationally accepted standards as well as International Atomic Energy Agency standards IAEA OH-1, IAEA OH-2, IAEA OH-3 and IAEA OH-4.

\section{Concentrating nanodiamonds from ice}

A detailed protocol is provided so other researchers can adequately test the results presented herein. Modified from Huss and Lewis (1995), this process is suitable for processing residue from ice samples that may contain NDs. This protocol is not recommended for terrestrial bulk sediment that contains NDs with a wide mix of other minerals that require more aggressive acid digestion. CAUTION: other standard ND extraction protocols are not recommended for extracting $\mathrm{n}$-diamonds.

\section{Supplies}

$50 \mathrm{~mL}$ centrifuge tubes (if necessary, substitute $15 \mathrm{~mL}$ tubes throughout); glass vials; beakers (Teflon or Pyrex-acidwashed); Teflon spatulas; analytical grade hydrochloric acid and ammonia. Pre-weigh all containers. All digestions/ extractions should be conducted in fume hoods when appropriate.

\section{Extracting the nanodiamonds}

Evaporation. Nearly all ND polytypes are metastable, except cubics, so do not exceed $100^{\circ} \mathrm{C}$ in any processing step. Optimal amount of ice or meltwater is $0.5-1 \mathrm{~L}$. Less ice decreases the potential for detecting NDs.

1. Measure weight and volume of ice or meltwater.

2. Place in a beaker and evaporate at $<100^{\circ} \mathrm{C}$. Take care to not overheat.

3. Thoroughly scrape out residue with a spatula and weigh.

Hydrochloric acid. Diamonds are naturally hydrophobic. However, they may become hydrophilic after long deposition due to addition of surface contaminants, which make them less susceptible to extraction. To remove possible contaminants:

1. Place a measured aliquot in a $50 \mathrm{~mL}$ centrifuge tube.

2. Fill with concentrated $\mathrm{HCl}$ at $\mathrm{pH}$ of $<1$.

3. Ultrasonicate for 1-2 hours.

4. Evaporate acid at $<100^{\circ} \mathrm{C}$ to $\sim 1-2 \mathrm{~mL}$. 
5. Recover the ND-rich residue by scraping the container with the appropriate spatulas.

Ammonia. In a neutral/alkaline environment NDs become colloidal, permitting efficient separation from most other minerals.

To place NDs into colloidal suspension:

1. Place residue in a beaker and fill with ammonia. The $\mathrm{pH}$ should be $>7$, with $\mathrm{pH}$ of 10-12 being preferable.

2. Sonicate for 1 hour at room temperature.

3. Heat in oven or on hotplate at $\sim 40-60^{\circ} \mathrm{C}$ for $24-48$ hours.

4. Remove from hotplate and sonicate for 5-10 min every 3-6 hours. Ensure the pH exceeds 7.

5. Centrifuge at $1000 \mathrm{~g}(1500 \mathrm{rpm})$ for 2 hours to separate out any suspended clay particles.

6. Transfer the supernatant to a $50 \mathrm{~mL}$ polypropylene centrifuge tube and start evaporating the solution at $100^{\circ} \mathrm{C}$.

7. Repeat steps 1-6 another one to five times, depending upon whether you wish to extract the maximum percentage of NDs.

8. Each time, add the resulting supernatant to the evaporating supernatant tube.

9. Reduce the final evaporating volume to $1-2 \mathrm{~mL}$. Cool.

Hydrochloric acid. Polar ice may contain substantial concentrations of ocean salts, which complicate TEM analyses. To both dilute the dissolved salts and to flocculate the suspended NDs:

1. Mix $\mathrm{HCl}$ with a supply of de-ionized water until a $\mathrm{pH}$ of $<0.5$ is reached.

2. Fill the centrifuge tube containing diamondiferous supernatant with the $\mathrm{HCl}$ mixture.

3. Sonicate for $30 \mathrm{~min}$.

4. Cap tubes and heat in oven or on hotplate at $\sim 40-60^{\circ} \mathrm{C}$ for 24-96 hours.

5. To condense flocculates, centrifuge at $1000 \mathrm{~g}$ ( $\sim 1500 \mathrm{rpm})$ for $\sim 1$ hour.

6. Using pipette, draw down most of supernatant, leaving 0.5-1 mL without disturbing the solid residue. Set supernatant aside.

7. Scrape tube with a spatula to concentrate residue.

8. Remove contents to a glass vial, washing tube with alcohol.

9. For more complete recovery, repeat steps 1-8 for supernatant.

10. Combine each ND-bearing separation.

11. Add enough ammonia to neutralize solution.

12. Dry to constant weight at $<100^{\circ} \mathrm{C}$.

Analyzing the nanodiamonds

The most stable TEM grids available are covered with carbononly films. Most other films are unsuitable because the electron beam can cause NDs to increase in temperature, causing the grid film to rupture. Grids with a 200- to 300count mesh work best. To reduce heating, it is best to keep TEM voltages in the 80-100 kV range, though higher voltages are suitable if the beam is kept dispersed rather than tightly focused. The same TEM grids can be used for SEM, STEM and HREM.

\section{Making a TEM grid}

1. To the vial of dry neutralized residue, add two to three drops of ammonium hydroxide $(\mathrm{pH}>12)$ to colloidally re-suspend any NDs.

2. Weigh the TEM grid before adding the solution.

3. Pipette one drop of this admixture onto the grid and then use the pipette to draw off most of the drop.

4. Dry the grid at room temperature.

5. Weigh the TEM grid after adding the solution and drying. This facilitates estimation of ND abundances.

6. Typically, at least $5-10 \%$ of the grid area should be randomly searched.

\section{Analytical microscope protocol}

For TEM work, we used JEOL 1200EX II and JEOL TEM 1210 transmission electron microscopes. Lower electron-beam voltages $(60-80 \mathrm{kV})$ were preferred because some ND polymorphs, such as n-diamonds and lonsdaleite, are metastable and may vaporize at the high temperatures generated at higher voltages. The TEMs were used to identify and image single and clustered crystals, and ND polymorphs were identified via SAED and FFT. Multiple measurements were taken across larger diamonds to determine composition. High-resolution TEM analysis was carried out in a FEI Titan ${ }^{\mathrm{TM}}$ 80-300 scanning transmission electron microscope (STEM) (FEl, Hillsboro, Oregon), which is equipped with an image corrector, high-angle annular dark field (HAADF) detector, EDS detector, Gatan imaging filter (GIF) and a 4-megapixel charge-coupled device (CCD) camera. The magnification of the microscope was calibrated using a standard cross-grating carbon replica (2160 lines $\mathrm{mm}^{-1}$ ) evaporated with gold. Chemical analysis was carried out using EDS and EELS. HREM and SAED patterns were used to determine the structure of the diamonds. Interplanar spacing measurement and FFT image processing were performed using Digital Micrograph software. EDS and EELS analysis demonstrated that the nanoparticles in HREM images contain only carbon.

\section{Estimated quantities}

ND concentrations (number and ppb) were estimated by calculating the starting weight of each bulk ice sample, weight of the residue after evaporation, weight of extract used per grid, average number of NDs in a representative area of a grid, percentage of non-diamonds encountered during testing and net percentage of the grid displaying NDs.

\section{Data storage}

All original data files were uploaded to the US National Snow and Ice Data Center (NSIDC). 